\title{
Tipping the balance between good and evil: aberrant 14-3-3 3 expression drives oncogenic TGF- $\beta$ signaling in metastatic breast cancers
}

\author{
Chevaun D. Morrison and William P. Schiemann ${ }^{*}$
}

\begin{abstract}
Transforming growth factor beta (TGF- $\beta$ ) readily suppresses the development of early-stage breast cancers, an activity that gives way to tumor promotion in their late-stage counterparts. The molecular mechanisms underlying this mysterious switch in TGF- $\beta$ function remain murky. In addressing this conundrum, Xu et al. observed aberrant 14-3-3 3 expression to prevent the formation of tumor-suppressive Smad2/ 3:p53 complexes, while simultaneously driving the generation of oncogenic Smad2/3:Gli2 complexes. Once formed, Smad2/3:Gli2 complexes stimulate the expression of parathyroid hormone-related protein necessary for breast cancer metastasis to bone. This viewpoint highlights $14-3-3 \zeta$ as an essential driver of oncogenic signaling by Smad2/3 and TGF- $\beta$ in metastatic breast cancers.
\end{abstract}

\section{Background}

Transforming growth factor beta (TGF- $\beta$ ) is a ubiquitously expressed cytokine that plays essential roles in regulating tissue development, differentiation, and homeostasis $[1,2]$. TGF- $\beta$ also functions as a prominent suppressor of mammary tumorigenesis, doing so by inhibiting mammary epithelial cell (MEC) proliferation and inducing their apoptosis $[1,2]$. The diverse cellular responses and anti-tumor activities of TGF- $\beta$ transpire in part through its "canonical" activation of the latent transcription factors Smad2 and Smad3, which interact with the transcriptional machinery to manage the transcriptome in a cell-specific and context-specific manner [3]. Interestingly, while early-stage breast cancers remain reliant upon canonical TGF- $\beta$ signaling and its tumor-suppressive

\footnotetext{
* Correspondence: wps20@case.edu

Case Comprehensive Cancer Center, Case Western Reserve University, Wolstein Research Building, 2103 Cornell Road, Cleveland, OH 44106, USA
}

activities, the progression and eventual metastasis of their late-stage counterparts depends upon the activation of noncanonical (i.e., Smad2/3-independent) TGF- $\beta$ effectors, including mitogen-activated protein (MAP) kinases, phosphoinositide 3-kinase (PI3K)/AKT, nuclear factor (NF)- $\mathrm{kB}$, and Yes-associated protein (YAP)/transcriptional co-activator with PDZ-binding motif (TAZ) $[4,5]$. The paradoxical switch in TGF- $\beta$ function during metastatic progression provided the impetus to develop targeted chemotherapies designed to inactivate the TGF- $\beta$ pathway and its oncogenic activities in late-stage carcinomas $[6,7]$. Unfortunately, anti-TGF- $\beta$ agents have exhibited limited success in clinical settings owing to an incomplete understanding of "how, when, and why" TGF- $\beta$ acquires oncogenic activity in metastatic settings. Equally perplexing is the extent to which the canonical and noncanonical TGF$\beta$ signaling systems contribute to metastatic progression. Thus, overcoming these knowledge gaps and defining the contextual clues whereby $\operatorname{Smad} 2 / 3$ signals coalesce with noncanonical effectors in driving oncogenic TGF- $\beta$ signaling will be essential to improving the efficacy of anti-TGF$\beta$ therapies.

\section{The article}

The 14-3-3 family of adapter molecules is comprised of seven members (i.e., 14-3-3 $\beta, 14-3-3 \gamma, 14-3-3 \varepsilon, 14-3-3 v$, $14-3-3 \sigma, 14-3-3 \tau$, and $14-3-3 \zeta$ ) that can function to either suppress or promote tumorigenesis, doing so by forming homodimeric and heterodimeric complexes that bind and sequester more than 200 phosphoproteins $[8,9]$. For instance, 14-3-3 $\sigma$ functions as a potent tumor suppressor through its ability to regulate p53 expression, an event frequently inactivated in breast cancers due to epigenetic silencing of 14-3-3 $\sigma$ expression [10, 11]. In stark contrast, aberrant $14-3-3 \zeta$ expression is readily observed in several human malignancies and predicts for poor prognoses in patients harboring breast, lung, or head and neck cancers [12]. Along these lines, elevated 14-3-3ל 
expression couples TGF- $\beta$ to the initiation of epithelialmesenchymal transition (EMT) and metastatic programs in mammary tumors [13], suggesting that dysregulated $14-3-3 \zeta$ expression and activity may play an essential role in regulating oncogenic TGF- $\beta$ signaling.

Indeed, in a provocative study published in Cancer

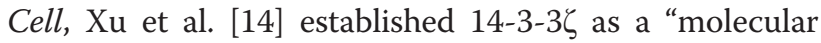
switch" that converts TGF- $\beta$ from acting as a tumor suppressor to a tumor promoter in metastatic breast cancers (Fig. 1). In doing so, the authors showed that the enforced expression of 14-3-3 $\zeta$ inactivates the tumorsuppressing functions of 14-3-3 $\sigma$, particularly its ability to stabilize p53 and coordinate the formation of Smad2/ 3:p53 complexes operant in driving p21 expression. Likewise, 14-3-3 $\zeta$ binds and sequesters phosphorylated YAP1 within the cytoplasm, thereby preventing its nuclear translocation and transactivation of 14-3-3 $\sigma$ expression. Collectively, these events coalesce to inactivate cytostatic TGF- $\beta$ signaling in premalignant MECs. Interestingly, rendering bone tropic breast cancer cells deficient in 14-3-3 $\zeta$ expression dramatically reduced their metastatic colonization and secondary outgrowth in the bones of mice. This cellular condition also prevented metastatic breast cancer cells from activating osteoclast maturation and bone osteolysis in response to TGF- $\beta$. Indeed, TGF- $\beta$-mediated bone metastasis required 14-3-3 $\zeta$ to stabilize Gli2 expression, leading to the formation of Smad2/3:Gli2 complexes that drive parathyroid hormonerelated protein (PHrT) expression. These findings are consistent with $14-3-3 \zeta$ expression being embedded in TGF- $\beta$ gene signatures coupled to breast cancer metastasis to bone, an event that significantly reduces bone metastasisfree survival in breast cancer patients. Collectively, this intriguing study establishes $14-3-3 \zeta$ as a preemptive molecule operant in inactivating the cytostatic functions of TGF- $\beta$ in premalignant MECs, and as an essential mediator that unifies canonical and noncanonical TGF- $\beta$ signaling outputs during breast cancer metastasis to bone.

\section{Viewpoint}

Overexpression of 14-3-3 $\zeta$ has been observed in numerous human cancers, including those of the esophagus, breast, lung, brain, and prostate $[12,15]$. More recently, $14-3-3 \zeta$ has been detected in the secretomes produced by these cancers, suggesting that monitoring 14-3-3 $\zeta$ expression may serve as a potential prognostic biomarker for late-stage breast carcinomas [9, 14]. Equally intriguing is the finding by $\mathrm{Xu}$ et al. that $14-3-3 \zeta$ is capable of dictating the expression patterns of additional 14-3-3

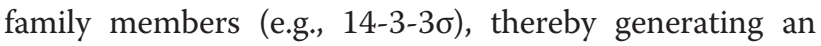
extensive level of control capable of fine-tuning the context and pathological output of metastatic signals stimulated by TGF- $\beta$. Accordingly, Boudreau et al. [16] demonstrated that 14-3-3 $\sigma$, which typically functions as a tumor suppressor, may in fact enhance the invasive behaviors of basal-like breast cancer cells, doing so by regulating cytoskeletal dynamics in a protein kinase $C_{\zeta}$ dependent manner. In both studies, aberrant 14-3-3 family member activity was associated with basal-like and triple-negative breast cancers (TNBCs), both of which respond to the tumor-promoting activities of TGF- $\beta$. It should be noted that basal-like/TNBCs are generally not highly metastatic to bone and instead preferentially metastasize to visceral organs and the brain [17], which contrasts sharply with their estrogen receptor alpha-positive counterparts that do in fact preferentially metastasize to bone and are responsive to the tumor-suppressing activities of TGF- $\beta[18,19]$. Future studies need to: (1) identify which specific basal-like/ TNBC subtypes are dependent upon dysregulated 14-33 family and oncogenic TGF- $\beta$ signaling; (2) establish the impact of aberrant 14-3-3 family member expression

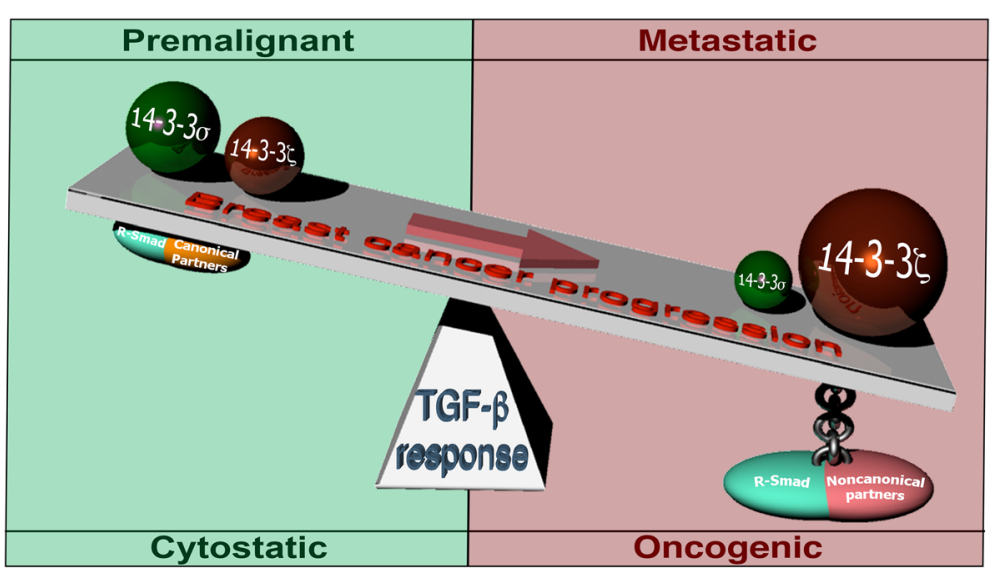

Fig. 1 Tipping the balance in transforming growth factor beta (TGF- $\beta$ ) signaling in metastatic breast cancers. Expression of 14-3-30 coordinates cytostatic TGF- $\beta$ signals in premalignant MECs, while 14-3-3 , expression and activity drives the acquisition of oncogenic TGF- $\beta$ signaling in metastatic breast cancer cells. See text for additional details 
in non-TNBC subtypes, as well as their response to TGF- $\beta$; and (3) demonstrate a causal role of dysregulated 14-3-3 family expression in dictating breast cancer dissemination to organ sites other than bone.

Finally, it is interesting to note that the activities associated with aberrant 14-3-3 $\zeta$ expression are highly reminiscent of those attributed to the oncogenic functions of TGF- $\beta$, including the ability to drive carcinoma cell proliferation, survival, and chemoresistance [1, 2, 9]. The highlighted work by $\mathrm{Xu}$ et al. implicates $14-3-3 \zeta$ as a prominent player that dictates the pathophysiology of canonical TGF- $\beta$ signaling in neoplastic cells. However, TGF- $\beta$ also activates numerous noncanonical signaling effectors that are equally essential in mediating the tumor-promoting functions of TGF- $\beta$ in developing and progressing breast cancers [4]. Future studies thus clearly need to expand our understanding of the role of 14-3-3 family members in eliciting metastatic progression driven by TGF- $\beta$, particularly with respect to determining the extent to which (1) aberrant 14-3-3 $\zeta$ expression engages the noncanonical TGF- $\beta$ signaling system, (2) noncanonical TGF- $\beta$ effectors engender dysregulated expression of 14-

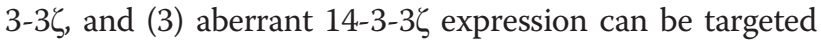
therapeutically and/or utilized as a predictive biomarker to delineate breast cancer patients most likely to respond to anti-TGF- $\beta$ agents. Ultimately, answering these and other questions will provide a foundation to develop more effective therapies against the oncogenic functions of TGF- $\beta$ and its stimulation of breast cancer metastasis.

\begin{abstract}
Abbreviations
EMT: Epithelial-mesenchymal transition; MAP: Mitogen-activated protein; MEC: Mammary epithelial cell; NF: Nuclear factor; PHrT: Parathyroid hormonerelated protein; PI3K: Phosphoinositide 3-kinase; TAZ: Transcriptional co-activator with PDZ-binding motif; TGF- $\beta$ : Transforming growth factor beta; TNBC: Triple-negative breast cancer; YAP: Yes-associated protein.
\end{abstract}

\section{Competing interests}

The authors declare that they have no competing interests.

\section{Acknowledgements}

Research support was provided in part by the National Institutes of Health to CDM (CA180670) and WPS (CA129359 and CA177069).

Published online: 11 July 2015

\section{References}

1. Tian M, Neil JR, Schiemann WP. Transforming growth factor- $\beta$ and the hallmarks of cancer. Cell Signal. 2011;23:951-62.

2. Massague J. TGF $\beta$ in cancer. Cell. 2008;134:215-30.

3. Massague J. TGFß signalling in context. Nat Rev Mol Cell Biol. 2012;13:616-30.

4. Parvani JG, Taylor MA, Schiemann WP. Noncanonical TGF- $\beta$ signaling during mammary tumorigenesis. J Mammary Gland Biol Neoplasia. 2011;16:127-46.

5. Barcellos-Hoff MH, Akhurst RJ. Transforming growth factor- $\beta$ in breast cancer: too much, too late. Breast Cancer Res. 2009;11:202.

6. Akhurst RJ, Hata A. Targeting the TGF $\beta$ signalling pathway in disease. Nat Rev Drug Discov. 2012;11:790-811.

7. Lee YH, Schiemann WP. Chemotherapeutic targeting of the transforming growth factor- $\beta$ pathway in breast cancers. Breast Cancer Manag. 2014;3:73-85.

8. Reinhardt HC, Yaffe MB. Phospho-Ser/Thr-binding domains: navigating the cell cycle and DNA damage response. Nat Rev Mol Cell Biol. 2013;14:563-80.
9. Matta A, Siu KW, Ralhan R. 14-3-3 $\zeta$ as novel molecular target for cancer therapy. Expert Opin Ther Targets. 2012;16:515-23.

10. Lee MH, Lozano G. Regulation of the p53-MDM2 pathway by 14-3-30 and other proteins. Semin Cancer Biol. 2006;16:225-34.

11. Ling C, Su VM, Zuo D, Muller WJ. Loss of the 14-3-30 tumor suppressor is a critical event in ErbB2-mediated tumor progression. Cancer Discov. 2012;2:68-81.

12. Neal CL, Yu D. 14-3-3 6 as a prognostic marker and therapeutic target for cancer. Expert Opin Ther Targets. 2010;14:1343-54.

13. Lu J, Guo H, Treekitkarnmongkol W, Li P, Zhang J, Shi B, et al. 14-3-3 cooperates with ErbB2 to promote ductal carcinoma in situ progression to invasive breast cancer by inducing epithelial-mesenchymal transition. Cancer Cell. 2009;16:195-207.

14. Xu J, Acharya S, Sahin O, Zhang Q, Saito Y, Yao J, et al. 14-3-3 $\zeta$ turns TGF- $\beta$ 's function from tumor suppressor to metastasis promoter in breast cancer by contextual changes of Smad partners from p53 to Gli2. Cancer Cell. 2015;27:177-92.

15. Li Y, Zou L, Li Q, Haibe-Kains B, Tian R, Li Y, et al. Amplification of LAPTM4B and $Y W H A Z$ contributes to chemotherapy resistance and recurrence of breast cancer. Nat Med. 2010;16:214-8.

16. Boudreau A, Tanner K, Wang D, Geyer FC, Reis-Filho JS, Bissell MJ. 14-3-30 stabilizes a complex of soluble actin and intermediate filament to enable breast tumor invasion. Proc Natl Acad Sci U S A. 2013;110:E3937-44.

17. Foulkes WD, Smith IE, Reis-Filho JS. Triple-negative breast cancer. N Engl J Med. 2010;363:1938-48.

18. Kennecke H, Yerushalmi R, Woods R, Cheang MC, Voduc D, Speers CH, et al. Metastatic behavior of breast cancer subtypes. J Clin Oncol. 2010;28:3271-7.

19. Moses H, Barcellos-Hoff MH. TGF- $\beta$ biology in mammary development and breast cancer. Cold Spring Harb Perspect Biol. 2011;3:a003277. 